\title{
DESDE EL RUIDO
}

\author{
Juan Andrés Crego Morán \\ Universidad del País Vasco / Euskal Herriko Unibertsitatea. Dpto. Arte y Tecnología
}

\section{Resumen}

En el Arte sonoro, o en el sonido usado dentro de una obra de arte, el ruido no debería observarse desde la perspectiva comunicacional, sino como un clase específica de sonido, que comporta significados en sí mismo. Partiendo de una aproximación terminológica se acotará el concepto de ruido según el sentido usual del término. Se analizará el impacto subjetivo sobre la percepción de un sonido como ruido. Mediante diversos ejemplos de obras de arte que incorporan el ruido se categorizarán según el comportamiento del ruido, propio de cada tipo. Si se acepta que el ruido no es una interferencia, puede desarrollarse como objeto y sujeto de experiencias estéticas.

Palabras-clave: ARTE; SONIDO; RUIDO; COMPLEJIDAD; CAOS

\section{FROM THE NOISE}

\section{Abstract}

Either in the Sound Art, or in the sound used in an artwork, the noise should not be observed from the communications perspective, but as a specific kind of sound that carries meaning itself. From a terminological approach the concept of noise will target on the usual sense. The subjective impact on the perception of sound as noise will be analyzed. Through various examples of artworks that incorporate noise they will be categorized according to the behavior of noise, specific to each type. If one accepts that noise is not an interference, it can develop as object and subject of aesthetic experiences.

Keywords:ART; SOUND; NOISE; COMPLEXITY; CHAOS

Crego Morán, Juan Andrés. 2015. “Desde el ruido”. AusArt 3 (2): 106-116. DOI: 10.1387/ausart.15942

\section{AUSART}




\section{DESDE EL RUIDO: DEFINICIÓN(ES)}

Sonido, rumor, rechinamiento, zumbido, crujido, chasquido, estruendo, fragor, estampido, detonación, estallido, estrépito, estridencia, alboroto, algarabía, griterío, barahúnda, escándalo, bulla, bullicio, etc. La sinonimia de ruido es muy extensa y variada. Y reveladora. En la selección precedente sólo se han tenido en cuenta aquellos sinónimos relacionados con el sentido primario y habitual de la palabra, aquél que dice "Sonido inarticulado, por lo general desagradable." (DRAE'1), o la similar definición "Sonido inarticulado y confuso más o menos fuerte." Estas definiciones dejan claro el reconocimiento de los ruidos como una clase particular de sonidos, así como una apreciación negativa del mismo. Nuestra intención en este texto es replantear el ruido desde una perspectiva propiamente artística que permita una resignificación positiva del mismo.

La procedencia etimológica de la palabra ruido sería del latín rugĭtus, que también da rugido. Pero la idea de algo inarticulado y molesto aparece también en otros idiomas. En inglés, por ejemplo, la palabra noise tiene también unas acepciones primarias muy similares a las de ruido y procedería a su vez del francés antiguo noise, una de cuyas etimologías posibles es del latín nausea. En el francés actual bruit significa "Manifestación sonora desagradable" ${ }^{3}$ y mantiene el mismo sentido primario que nuestro ruido, y mantiene también relación etimológica con el latín rugĭtus. En un breve artículo publicado en Mediateletipos sobre la palabra ruido en diversos idiomas, Blanca Rego dice:

“Llama la atención que en las lenguas occidentales el ruido parezca provenir casi siempre de una amenaza o alteración exterior, mientras que en las orientales da la impresión de que la alteración provenga de nosotros mismos."

(Rego 2014)

Obviamente se trata de las acepciones primarias, quedando descartadas por tanto segundas y terceras acepciones, que suelen ser en realidad derivaciones semánticas de la primaria. De ser necesario, sin embargo, se recurrirá puntualmente a definiciones más técnicas. 


\section{RUIDO 0 NO RUIDO}

El ruido, los ruidos, molestan particularmente si son muy intensos, al extremo de que un sonido que no molesta inicialmente empieza a hacerlo según aumenta su intensidad. Porque hay una frontera traspasada la cual un sonido, sin dejar de serlo, se percibe ya como ruido. Esta frontera es personal, circunstancial y cultural, lo que equivale a decir que el ruido mismo es un concepto personal, circunstancial y cultural. Para un fanático del heavy metal el volumen sonoro elevado por los amplificadores es parte misma de la música que disfruta. No obstante, sobrepasar ciertos umbrales para elevadas intensidades sonoras puede ser no sólo doloroso, sino destructivo. ${ }^{4}$

Un ruido de muy baja intensidad es percibido de diferente forma. Partiendo de que todo ruido no deja de ser un sonido, aquí pueden ambos ser sinónimos, pues puede no generar molestia ni, por tanto, rechazo. Dominaría entonces la percepción del ruido como sonido inarticulado, que no llega a desarrollar un código semejante al del habla o al musical. Carraspeos y crujidos son característicos sonidos/ruidos, entre muchos otros, que raramente son molestos. De hecho también son portadores de significado, a pesar de ser inarticulados, puesto que informan de sucesos que acontecen en el entorno. La ausencia de codificación no supone ausencia de información. Por otra parte la percepción de un ruido como fuerte o débil no depende sólo de su propia intensidad, sino que influye de manera importante la intensidad del marco sonoro que le rodea, pudiendo parecer débil rodeado de sonidos más fuertes y viceversa. Pero un sonido débil, en principio no molesto, puede transformarse en ruido molesto con sólo que se repita constantemente o con demasiada frecuencia. La percepción de la intensidad de un ruido es relativa, además de subjetiva.

\section{EL RUIDO-SONIDO}

"With Hidden Noise" es una de las más misteriosas piezas de Marcel Duchamp, categorizada por el propio autor como un "ready-made ayudado". Originalmente fue realizado en Nueva York en 1916, aunque existen varias réplicas realizadas bajo la supervisión de Duchamp. En la descripción de la pieza incluida en el catálogo del Philadelphia Museum of Art ${ }^{5}$ se nos presenta como "Madeja de cuerda entre dos planchas de bronce, unidas por cuatro tornillos largos, que contiene objeto desconocido añadido por Walter Arensberg". 
Resulta algo extraño que esta descripción no haga referencia al sonido, que da título a la pieza. El comentario de la misma, por el contrario, sí que alude al origen del título:

"Luego pidió a su amigo y mecenas Walter Arensberg que colocase un objeto desconocido en el interior antes de que cerrase el Readymade con cuatro tornillos largos. El título alude al golpeteo que el objeto oculto hace cuando se agita. Duchamp solicitó a Arensberg que nunca le dijera qué era el objeto secreto, prefiriendo permanecer felizmente ignorante del "contenido" de su obra." 6

(Temkin, Rosenberg, y Taylor 2000)

En esta obra de Duchamp el sonido producido por un objeto desconocido en su interior, en sí mismo un ruido indeterminado, se ve además tamizado por el ovillo, que lo apaga o modifica, pero ¿puede decirse que lo interfiere o más bien que lo conforma? Este ruido-sonido, de poca intensidad y ademas ocasional como para ser molesto, es la clave misma de la pieza, a pesar de que sólo aparece al sacudirla. Lo que conduce a una situación paradójica, demasiado frecuente en los museos, que nos impide acceder el sonido pues no está permitido tocar, ni menos aún sacudir, las obras. Si se hace una ejercicio de imaginación es posible aproximarse al sentido de esta pieza, pues resulta obvio que una agitación suave produciría ruidos suaves también, mientras que fuertes sacudidas incrementarían en número e intensidad los ruidos aleatorios producidos por el objeto en su interior ${ }^{7}$.

\section{EL RUIDO MOLESTO}

De acuerdo con la acepción habitual de ruido como un sonido molesto, cabría pensar que debería evitarse esta circunstancia dentro de una obra de arte, pero esto sería una presunción ingenua. En realidad puede ser una herramienta extremadamente útil para causar un efecto intenso e incómodo sobre el espectador. Wolf Vostell emplea el ruido así en la escultura ambiente "Fiebre del Automóvil" (1973) ${ }^{8}$, donde periódicamente entran en funcionamiento unos rastrillos metálicos que producen un intenso, inquietante y molesto rechinamiento al moverse adelante y atrás sobre unas planchas metálicas. 
Se reúnen aquí casi todos los elementos que convierten al ruido en un molesto sonido, tal como se mencionó en un apartado anterior. El sonido por sí mismo es desagradable e irritante, como lo suele ser cualquier rechinar (de dientes, de uñas sobre una pizarra), pero se añade la intensidad producto de la multiplicación por el total de seis rastrillos que surgen del vehículo. La pieza se pone en marcha periódicamente, pero de estar en funcionamiento constante se tendría que sumar como factor irritante la permanencia del ruido, que se convertiría en insoportable, y que probablemente provocaría la deserción del público. Otro factor que se añade es el ruido que producen unos martillos sobre la chapa del coche, más retumbante y aleatorio que el de los rastrillos. La combinación de ambos ruidos genera una sobrecarga sensorial que es un factor determinante en el impacto de la obra sobre el espectador.

Vostell desarrolló "Fiebre del Automóvil" a partir de un happening que presentó en Berlín ese mismo año, con el título "Berlin-Fieber" (Lozano Bartolozzi 2000; Uelsberg 2007), mostrando como el artista replantea el mismo tema en dos obras de naturaleza diferente, viéndose obligado a recurrir en la obra expositiva al uso del elemento sonoro para reintroducir de alguna forma el ambiente característico de sus happenings de la época. La misma denominación de escultura ambiente deja claro dicho interés, y no cabe duda de que la forma de recrear la intensidad ambiental del happening reside aquí en el intenso y enervante ruido que emite la instalación. El espectador puede no mirar la pieza, si así lo decide, pero el ruido que ésta produce llena el espacio en que se ubica y el espectador no puede evitar verse envuelto por él, con lo que Vostell consigue recrear el efecto ambiental además de provocar en los espectadores-auditores una sensación intensa de incomodidad, que refuerza el tema de la obra.

"La preocupación de Wolf Vostell era establecer un diálogo dirigido entre arte y vida cotidiana en transición para evocar procesos que convierten la acción y la observación al mismo tiempo en un proceso de cambio mental y existencial. Vostell lo formulaba como preguntas acerca de la sociedad y entorno de la gente, que está siempre amenazada por la violencia, la intolerancia, la guerra y la muerte." 9 


\section{RUIDO Y MÚSICA}

Hablar de la presencia del ruido en el arte supone inevitablemente hablar de la presencia del ruido en la música. Aunque la relación de la música y el ruido requeriría una visión extensa y detallada, excedería del objetivo del presente texto, por lo que se plantearán sólo dos alternativas basadas en el modo en que ambos conceptos se imbrican: el ruido como materia musical en sí y la música que se sirve del ruido.

\section{RUIDO MUSICAL}

Todo ruido no es otra cosa que sonido. A lo largo de la evolución humana inició su desarrollo lo que hoy conocemos como música, pues por diferentes formas que adopte en momentos y culturas diferentes siempre es reconocible como tal. Obviamente tuvo que comenzar de un modo simple, pero que al menos conectase varios sonidos en una sucesión intencional (Arom 2001). Presumiblemente serían sonidos vocales o percusiones con materiales casuales, en cualquier caso no se han encontrado evidencia de tiempos remotos y todo se reduce al terreno hipotético. Las percusiones son algo que podemos caracterizar como ruidos, aunque con el tiempo la aparición de elementos capaces de generar sonido por procedimientos diferentes a la percusión fueron refinándose hasta producir sonidos depurados, con frecuencias sonoras dominantes que ya son difíciles de clasificar como ruidos desestructurados.

En cierto modo los ruidos que existieron en el origen de la música fueron desplazados casi completamente de las tradiciones musicales más desarrolladas. En occidente hubo que esperar al Futurismo para que el ruido fuera redescubierto como materia musical. Las bases teóricas fueron formuladas en marzo de 1913 por Luigi Russolo en una carta-manifiesto, más tarde publicada como libro, en 1916. En "L'Arte dei rumori" expone entre otros aspectos las 6 familias de ruidos de la orquesta futurista. A modo de referencia se mencionan a continuación uno de cada familia: Estruendos, Silbidos, Murmullos, Chirridos, Ruidos de percusión y Voces humanas y animales (Russolo 1998). Puede verse que se relacionan fácilmente con la lista de sinónimos de ruido con la que se dio inicio a este texto, aunque en muchos casos podríamos decir que se trata más bien de hipónimos ${ }^{10}$ que de sinónimos. 
Según Russolo nos dice en su primera conclusión:

"Los músicos futuristas deben ampliar y enriquecer cada vez más el campo de los sonidos. Esto responde a una necesidad de nuestra sensibilidad. De hecho, en los compositores geniales de hoy notamos una tendencia hacia las más complicadas disonancias. Al apartarse progresivamente del sonido puro, casi alcanzan el sonido-ruido. Esta necesidad y esta tendencia no podrán ser satisfechas sino añadiendo y sustituyendo los sonidos por los ruidos."

(Russolo 1998)

La musicalización del ruido no consiste en tomar y refinar los ruidos para que pierdan su rudeza, sino por el contrario aceptarlos como tales y crear música con ellos. Una música muy diferente de la habitual, por supuesto, teniendo en cuenta que los ruidos a los que Russolo alude son producidos por máquinas principalmente mecánicas

"Así, los motores y las máquinas de nuestras ciudades industriales podrán un día ser sabiamente entonados, con el fin de hacer de cada fábrica una embriagadora orquesta de ruidos."

(Russolo 1998)

Que las máquinas actuales sean muchas silenciosas o produzcan sonidos no propios, sino sintéticos o externos a ellas, podría llevar a que para realizar el programa de Russolo fuera imprescindible recurrir a máquinas antiguas o pseudomáquinas como los instrumentos que desarrolló en su día, los "intonarumori”. O bien podría llevar a un tipo de música de los ruidos muy diferente de la que el imaginaba, como quizá sea el caso de la música noise de Merzbow ${ }^{11}$.

\section{MÚSICA RUIDOSA}

En esta segunda aproximación es la música la que gradualmente se ruidifica, es decir, que va incorporando elementos que desde un punto de vista convencional no son sino ruidos. Obviamente no es una aproximación realmente ajena a la previa, ni mucho menos opuesta, sino un mecanismo alternativo de valorización del ruido en el ámbito musical. Aquí domina el factor distorsionante, aquel que introduce voluntariamente elementos que deterioran el 
sonido musical. Decir que lo deterioran es partir de una posición técnica, no artística, pues la clave es entender que mediante una operación estética el artista puede transformar el ruido en algo aceptable. O mejor dicho, aceptable para según qué público. Un público culto y entrenado podía captar y entender las piezas de piano preparado de John Cage, pero la situación era muy distinta en la música popular. Cuando en la década de 1950 varios músicos (Willie Kizart, Chet Atkins) de una manera accidental generaron sonidos distorsionados con la amplificación defectuosa de sus guitarras es difícil que previeran el incremento y asentamiento, e incluso la comercialización del (d)efecto en las décadas subsiguientes. En ciertos ámbitos específicos como el noise rock llegó a convertirse en un cliché, que aparece también sistemáticamente en muchos temas de heavy metal, en cualquiera de sus manifestaciones.

La proliferación de variantes de efectos distorsionantes, primero analógicos y luego digitales, ha ido dando carta de naturaleza al ruido dentro de la música en un amplio rango de variantes. Al mismo tiempo su público ha evolucionado con ello, adaptándose a interpretar como un elemento musical más el ruido que aparece superpuesto, e incluso excluyente por momentos, a los elementos musicales convencionales. Este extenso auditorio ha asimilado el ruido como un carácter estético, lo que puede asimilarse a planteamientos de Umberto Eco (2007) en la "Historia de la fealdad", donde se revela que ha sido constante una apreciación estética de lo feo. Menciona Eco brevemente que el compositor Louis Spohr dijo que era "una orgía de estruendo y de vulgaridad", tras escuchar la primera audición pública de la "Quinta Sinfonía" de Beethoven. Quizá fuese un caso similar que en la música antigua occidental debiera evitarse el intervalo de tritono, llamado también diabolus in musica, por considerarse un sonido conflictivo, con sonoridades diabólicas, difícil de aceptar durante mucho tiempo y visto en cierto modo como ruido. Pasado el tiempo muchos sonidos inquietantes o molestos han ido siendo integrados $y$ aceptados, en un proceso que parece irreversible.

La pregunta entonces sería, en palabras de Paul Hegarty:

“En qué punto pierde el ruido su ruidosidad y se convierte en sentido, música, significación? ¿ ¿existe incluso un punto en el que el ruido puede subsistir?"

(Hegarty 2001) 


\section{EL RUIDO COMO SUMA Y ORIGEN DE TODO}

O de casi todo, pues la diferencia no es tan significativa. Cualquiera de las veces que se ha programado la pieza 4'33" desde que John Cage la compusiese en 1952, se ha creado un espacio-tiempo de silencio teórico (Wallin, Merker y Brown 2001), pero de no silencio efectivo. Pues es sabido que incluso en situaciones que no se generan ruidos voluntarios es suficiente ruido el que se genera involuntariamente. Al menos la primera vez que la pieza se interpretó, pues probablemente el resto de la ocasiones haya habido más ruidos escenificados de los que debería haber. En dicha primera e inalcanzable actuación un grupo de asistentes desprevenidos desarrollaron una cacofonía de ruidos de todo tipo y variadas intensidades, absolutamente irrepetibles. Intérpretes y a la vez oyentes de una pieza que muchos no escucharían aunque la estuviesen oyendo. Si fuesen 10, 100 o 1000 personas las asistentes el efecto global cambiaría progresivamente, incluso aunque el tipo de ruidos producidos fuesen similares. Con un incremento suficiente se iría aproximando al ruido blanco.

Si los ruidos individuales y discretos pueden ser fácilmente reconocibles por sus características sonoras, este ruido blanco o, quizá sea mejor llamarlo gris $^{12}$, sería casi perfectamente uniforme e imprecisable. Y sólo sería necesario sumar un sinnúmero de ruidos individuales para ello. Gris, porque un oyente ubicado en un entorno amplio o muy amplio que pudiese estar escuchando literalmente decenas o centenas de miles de sonidos superpuestos, para él todos serían muy débiles por la distancia a que estuviesen y la suma de todos ellos aun siendo notoria no llegaría a ser demasiado intensa. El resultado sería algo opaco, gris, como el fieltro que usaba Joseph Beuys ${ }^{13}$. Gris, como cuando se está en medio de una niebla intensa, que no permite ver nada a sólo unos metros de distancia, como los sonidos lejanos que llegan a través de una niebla densa.

Sin embargo ese ruido gris tiene una vibración porque es la suma de una infinidad de ruidos que no son constantes, por lo que desde una casi imperceptible variación ocasional destaca ligeramente algún sonido individual, pues idealmente si contiene todos los ruidos, de él pueden salir todos ellos. 


\section{CONCLUSIÓN}

El ruido es un fenómeno complejo, susceptible de diversas aproximaciones, tal y como se ha visto en los aparados precedentes. Aunque el fenómeno admite una interpretación científico-técnica que permite cuantificar determinados parámetros, el punto de vista expuesto sostiene que un sonido se percibirá como un ruido agresivo o molesto dependiendo de las circunstancias fundamentalmente. Pero una parte muy importante de dichas circunstancias será el propio oyente pues su actitud y expectativas condicionarán su percepción cómo ruido indeseado o, por el contrario, como sonidos que le rodean y que pueden ser aceptables, entendibles e incluso disfrutables. Desde el arte el ruido debería ser asumido en todas sus modalidades y circunstancias, molestas y placenteras, utilizándolo como una materia prima de la que se extraiga todo lo que se precise.

\section{Referencias}

Arom, Simha. 2001. "Prolegomena to a Biomusicology". En The Origins of Music, Nils Lennart Wallin, Björn Merker, and Steven Brown, eds., 27-9. Cambridge, MA: MIT

Eco, Umberto, ed. 2007. Historia de la fealdad. Traducido por María Pons Irazazábal. BarceIona: Lumen

Hainge, Greg. 2013. Noise matters: Towards an Ontology of Noise. New York: Bloomsbury

Hegarty, Paul. 2001. "Full with Noise: Theory and Japanese Noise Music". Ctheory (8 de noviembre). http://www.ctheory.net/articles.aspx?id=314

Kroker, Arthur \& Marilouise Kroker, eds. 2004. Life in the Wires: The CTheory Reader. Montréal: New World Perspectives

Lozano Bartolozzi, María del Mar. 2000. Wolf Vostell (1932-1998). Arte Hoy 6. Madrid: Nerea

Rego Constela, Blanca. 2014. "Etimología del ruido". Mediateletipos (blog). 16 de julio. http:// www.mediateletipos.net/archives/29414

Russolo, Luigi. 1998. El arte de los ruidos. Traducido por Olga Alas y Leopoldo Alas. Cuenca: Centro de Creación Experimental

Temkin, Ann, Susan Rosenberg and Michael Taylor. 2000. Twentieth Century Painting and Sculpture in the Philadelphia Museum of Art. Phildelphia Museum of Art

Uelsberg, Gabriele. (2007) 2014. "Wolf Vostell, Berlin-Fieber". Infotexte (blog). 16 de enero. https://infotexte.wordpress.com/2014/01/16/wolf-vostell-berlin-fieber-text-von-gabriele-uelsberg-2007/

Vostell, Wolf. 2007. Meine Kunst ist der ewige Widerstand gegen den Tod [My art is the eternal resistance to death]. Catálogo de la exposición. Gabriele Uelsberg, ed. Bonn: Rheinisches Landesmuseum 
Vostell, Wolf et al. 2003. Museo Vostell Malpartida: Colección Wolf y Mercedes Vostell. [Catálogo de la exposición]. Mérida: Editora Regional de Extremadura

Wallin, Nils Lennart, Björn Merker, and Steven Brown, eds. 2001a. The Origins of Music. Cambridge, MA: MIT

- 2001b. "An introduction to Evolutionary Musicology". En The Origins of Music, 3-24. Cambridge, MA: MIT

Notas

${ }^{1}$ RAE, Diccionario de la lengua española. $23^{a}$ ed., ed. del Tricentenario 2014, s.v. "ruido". http:// dle.rae.es/?id=WoW1aWq

${ }^{2}$ WordReference, s.v. "ruido". http://www.wordreference.com/definicion/ruido

3 "Manifestation sonore déplaisante". En Wiktionnaire, le dictionnaire libre, s.v. "bruit". https:// fr.wiktionary.org/wiki/bruit

${ }^{4}$ El oyente medio experimenta malestar significativo en un campo libre a niveles de presión sonora por encima de $120 \mathrm{~dB}$. A un nivel de aproximadamente $140 \mathrm{~dB}$, el malestar alcanza el punto de dolor. http://www.elruido.com/portal/web/guest/umbrales-auditivos

${ }^{5}$ http://www.philamuseum.org/collections/permanent/51541.html

${ }^{6}$ Traducción propia. La cita original es "He then asked his friend and patron Walter Arensberg to place an unknown object inside before he clamped the Readymade shut with four long screws. The title alludes to the rattling sound the hidden object makes when shaken. Duchamp requested that Arensberg never tell him what the secret thing was, preferring to remain blissfully ignorant of his work's "content»".

${ }^{7}$ Una ardua búsqueda de registro sonoro de esta pieza no ha producido resultados.

${ }^{8}$ Auto-Fieber - Fiebre del Automóvil, 1973. Escultura ambiente. Cadillac-Sedan, 8 motores, hoz, diez martillos grandes, seis rastrillos, pelos, dos torsos en plomo y mil platos.

https://www.youtube.com/watch?v=l-RkU7U0-tk

${ }^{9}$ Es traducción propia. Este es el texto original en Alemán: "Wolf Vostell ging es darum, einen gezielten Dialog von Kunst und alltäglichem Leben in Gang zu setzen, um Prozesse zu evozieren, die Handlung und Beobachtung gleichzeitig in einen gedanklichen und existentiellen Veränderungsprozess überführen. Vostell formulierte so Fragen über Gesellschaft und Umfeld des Menschen, der immer wieder durch Gewalt, Intoleranz, Krieg und Tod bedroht ist" (Uelsberg 2007).

${ }^{10}$ Pues palabras como crujido, griterío o estruendo son más específicas que ruido, pero mantienen las mismas características semánticas que esta última.

${ }^{11}$ Merzbow es el alias del japonés Masami Akita, un homenaje a Dadá y a Merzbau (suena muy similar).

${ }^{12}$ Que no debe entenderse en el sentido de un ruido cuya densidad espectral de potencia corresponde al nivel de presión sonora que debería tener cada frecuencia para que todas fuesen percibidas con la misma intensidad aparente.

${ }^{13}$ Joseph Beuys, Plight, 1958/1985, instalación: 43 rollos de fieltro, piano, mesa negra, termómetro, Centre Georges Pompidou, Paris. 